\title{
Zonificación forestal para Especies Multipropósito en la cuenca alta del río Bogotá. Estudio de caso: Villapinzón
}

Fernando Rodríguez Mojica*

Resumen: En el artículo se presentan los resultados más importantes de la inves tigación realizada en una región de la Cuenca Alta del río Bogotá, con el objeto de localizar las áreas que ofrecen mayor aptitud biofísica y socioeconómica para el esta blecimiento de especies forestales multipropósito, enmarcada dentro de la concepción de desarrollo sostenible.

Con este propósito se utilizó el esquema de Evaluación de Tierras propuesto por la FAO, el cual permite determinar la adaptabilidad de diferentes tipos de tierra para un tipo de uso seleccionado, representada espacialmente mediante el proceso de zonificación donde se agrupan las áreas con similares condiciones de aptitud.

Los resultados obtenidos muestran que en la región existe un importante número de unidades ambientales con buena aptitud biofísica para el establecimiento de las espe cies Laurel de cera y Uva camarona, pero la inclusión de parámetros socioeconómicos como el nivel de escolaridad y el acceso al crédito, reducen de manera significativa la aptitud integral de dichas tierras.

Palabras clave: Evaluación de tierras, Zonificación, Grado de aptitud, Aspectos biofísicos, Aspectos socioeconómicos.

(Aceptado noviembre 2002).

\footnotetext{
* Ingeniero Forestal, Universidad Distrital Francisco José de Caldas, Bogotá. Magíster en Geografía, énfasis en Ordenamiento Territorial, EPG, Convenio UPTC-IGAC, Bogotá.
} 


\section{Introducción}

El espacio geográfico como expresión dinámica de las relaciones entre el ambiente natural y la acción humana, ha estado en permanente transformación desde la misma aparición del hombre sobre la tierra como respuesta a la necesidad de encontrar el alimento y el bienestar, requeridos para alcanzar mejores condiciones de vida tanto individual como colectivamente.

Como producto de esta situación, actualmente se encuentran diversas formas de ocupación del espacio geográfico, localizadas generalmente en sitios donde las condiciones ofrecen las mayores posibilidades, aunque sin el conocimiento real de la verdadera oferta socioambiental del medio, asociada con los requerimientos de los tipos de utilización, por lo que se termina escogiendo actividades que aunque aparentemente son más productivas, resultan de alguna manera más impactantes sobre el entorno natural.

En esta relación dada por la oferta del medio (cualidades) y la demanda de los tipos de utilización (requerimientos), se hace necesario entender que las diversas unidades de tierra consideradas, presentan diferentes respuestas a las exigencias de las plantas, debido a la presencia de ciertas características del medio físico, razón por la cual las opciones de uso de dichos espacios, deben ser analizadas de forma particular y específica para ciertos tipos de utilización, que finalmente representen la mejor alternativa de desarrollo para la población.

Sin embargo, los sistemas utilizados actualmente para la determinación de la capacidad de uso de la tierra se enfocan hacia el establecimiento de usos generales, sin especificación alguna acerca de un uso particular y sin considerar la tierra como un concepto integral, donde se involucre el ambiente físico en todas sus manifestaciones, en asocio con las diferentes acciones de orden socioeconómico, que permitan la toma de decisiones más apropiadas en relación con la mejor ocupación, utilización y transformación del territorio.

Dada la poca experiencia existente en el país en esta materia y la imperiosa necesidad de avanzar hacia el uso masivo y comprensión de estos esquemas metodológicos, se adelantó la presente investigación que, al tiempo de buscar nuevas opciones de producción para una región tan importante como la Cuenca Alta del río Bogotá, buscó privilegiar la inclusión de los recursos forestales multipropósito como alternativa de desarrollo sostenible.

Con base en esto, se propuso determinar y representar espacialmente la aptitud biofísica de las unidades de tierra correspondientes a los municipios de Chocontá, Cogua, Guasca, Guatavita, Nemocón, Sesquilé, Suesca y Villapinzón (ver mapa 1), con el propósito de establecer plantaciones

32 Fernando Rodríguez Mojica 
comerciales de Laurel de cera y Uva camarona.

Consciente de la abstracción del componente socioeconómico que generalmente se ha observado en la mayor parte de los estudios relacionados con la evaluación de tierras, y dada la dificultad de contar con una información sistemática y suficiente para adelantar un análisis integral, contemplando aspectos biofísicos y socioeconómicos para toda la región, se tomó como estudio de caso el municipio de Villapinzón dada la posibilidad de contar con la información necesaria, difícil de tener para los demás municipios, y así tratar de llevar a cabo lo establecido por la propuesta metodológica de la FAO para este tipo de estudios.

Finalmente, se debe precisar que la metodología aquí empleada constituye una primera aproximación tanto para el análisis de aspectos forestales, como para la evaluación integral de un territorio, sujeta a ser ajustada y mejorada en la medida que se adelanten nuevas aplicaciones. Su aporte más importante se relaciona con el análisis de variables socioeconómicas como condición metodológica para evaluar la aptitud integral de tierras con fines productivos.

\section{Marco conceptual}

En su concepción más general, el ordenamiento territorial se concibe como una política encaminada a intervenir y modificar la organización espacial de un territorio, orientar su ocupación y optimizar su aprovechamiento, teniendo en cuenta las potencialidades y limitaciones del medio biofísico, socioeconómico, cultural y políticoadministrativo, enmarcado dentro de una perspectiva sustentable, dirigida a mejorar la calidad de vida de la población, en armonía con la conservación del ambiente. Esta situación plantea la necesidad de proponer nuevas alternativas de ocupación del territorio a partir de una distribución ordenada de actividades, para lo cual se debe considerar la planificación del uso de la tierra como el proceso por medio del cual se seleccionan las formas óptimas para utilizar la tierra teniendo en cuenta la realidad biofísica, tecnológica, social, económica y política de una región en particular (Andrade, 1996).

Para este propósito la Evaluación de Tierras se constituye en una actividad clave, dado que su objetivo fundamental se centra en la posibilidad de seleccionar formas óptimas de uso para cada unidad de tierra, considerando aspectos biofísicos, socioeconómicos y técnicos, promoviendo la conservación de los recursos con el propósito de poner a disposición del agricultor, planificador y de las instituciones, la información adecuada, referente al recurso tierra necesaria para adoptar decisiones de planificación, desarrollo y ordenación del espacio geográfico (FAO, 1985). 
Esta propuesta metodológica persigue como objetivo fundamental la aplicación de un sistema que permite identificar el mejor uso posible de las diferentes unidades de tierra a partir de una clasificación de la aptitud de la misma, para un tipo específico de uso, para lo cual el uso de la tierra es tan determinante como la tierra misma.

En este contexto se debe considerar que cada tipo específico de uso de la tierra tiene una serie de condiciones socioambientales favorables o adversas, las cuales corresponden a los requerimientos necesarios o deseables para la práctica exitosa y sostenida de un tipo de utilización y corresponden a la demanda de ciertos atributos que los tipos de utilización requieren, los cuales aseguran un comportamiento óptimo de la tierra y por consiguiente una buena respuesta del tipo de utilización a esas condiciones.

De igual manera, el componente que se relaciona con la tierra en sí misma comprende dos aspectos; el primero tiene que ver con la determinación de las unidades de tierra, el cual corresponde a la parte espacial de la evaluación y el segundo, con la selección de los factores diagnósticos representados por las cualidades y características de cada unidad de tierra, considerando tanto los aspectos biofísicos como socioeconómicos.

A partir de esta información se hace necesario precisar el grado de adaptabilidad de los tipos de utilización se- leccionados en las diferentes unidades de tierra delimitadas, definiendo así la aptitud de uso, considerada como el grado de intensidad con que las limitaciones de una unidad de tierra afectan el uso de la misma en forma general o particular, con base en el acercamiento entre las cualidades de la tierra y los requerimientos de los tipos de utilización propuestos (FAO, 1984).

Finalmente, se debe establecer un marco integrador referenciado espacialmente, para lo cual hay que adelantar una separación de la región en sus áreas componentes mediante un proceso de zonificación, cuyo grado de homogeneidad constituye su característica más importante ya que esto facilita cierta uniformidad en el comportamiento de las variables consideradas en relación con el nivel de estudio, permitiendo una mayor coherencia en las decisiones a tomar, respecto a la mejor utilización a partir de sus condiciones naturales y sociales específicas.

\section{Metodología}

La metodología de Zonificación Forestal aquí planteada, se desarrolló bajo una perspectiva descriptiva, con base en la guía sobre Evaluación de Tierras con fines Forestales propuesta por la FAO en 1984, con el propósito de determinar la adaptabilidad o idoneidad de la tierra para el establecimiento de actividades forestales, a partir de la evaluación y agrupamien-

34 Fernando Rodríguez Mojica 
to de zonas específicas de tierra en función de su aptitud para usos específicos, mediante la confrontación analítica de las características de la tierra, expresadas como cualidades, con las exigencias de los tipos de utilización, expresadas como requerimientos.

Básicamente se trata de establecer si una oferta de la unidad de tierra satisface los requerimientos del tipo de uso, para lo cual se debe partir del análisis integrado de los aspectos biofísicos y socioeconómicos, cuya secuencia se presenta en el esquema metodológico que aparece en la figura 1.

TIPOS DE UTILIZACIÓN DE LA TIERRA: la alternativa de uso propuesta se relaciona con actividades forestales considerando especies multipropósito, capaces de proporcionar de manera sostenible diversas posibilidades en cuanto a sus usos y valores, con beneficios que repercuten directamente en el bienestar de la población en plazos relativamente cortos, particularmente de productos no maderables de gran valor como materia prima en la elaboración de productos agroindustriales, así como también en la recuperación de áreas degradadas y conservación de recursos de gran valor social y económico como el agua y los suelos.

Para tal efecto se seleccionaron las especies laurel de cera y uva camarona como los tipos de uso específico, teniendo en cuenta el gran potencial económico y ecológico que ellas repre- sentan con base en el conocimiento de sus bondades como especies promisorias de gran valor, para ser comercialmente aprovechadas en esta región geográfica.

DEFINICION DE LAS UNIDADES DE TIERRA: en la definición de las unidades de tierra se tuvieron en cuenta dos aspectos: el levantamiento de las unidades de tierra que representa la parte espacial de la evaluación, y la selección de los factores diagnósticos relacionados con las características y cualidades de cada unidad de terreno, las cuales conforman la base de comparación con los requerimientos del tipo de uso de la tierra.

ANALISIS BIOFISICO: las unidades de tierra se delimitaron tomando como base el proceso de zonificación ecológica, con el propósito de obtener un análisis-síntesis integrado del paisaje, tratando de lograr una homogeneidad ambiental capaz de alcanzar el mejor comportamiento frente a los diferentes tipos de uso forestal, considerando los factores físicos de mayor incidencia en el uso de la tierra, en su manejo, crecimiento y productividad.

Los factores seleccionados tienen que ver principalmente con aspectos relacionados con el clima, la geoforma, el relieve, los suelos, la cobertura y uso de la tierra, los cuales tratados de manera integral mediante el uso de imágenes de percepción remota originaron las unidades biofísicas de tierra, cuya información se tomó del es- 


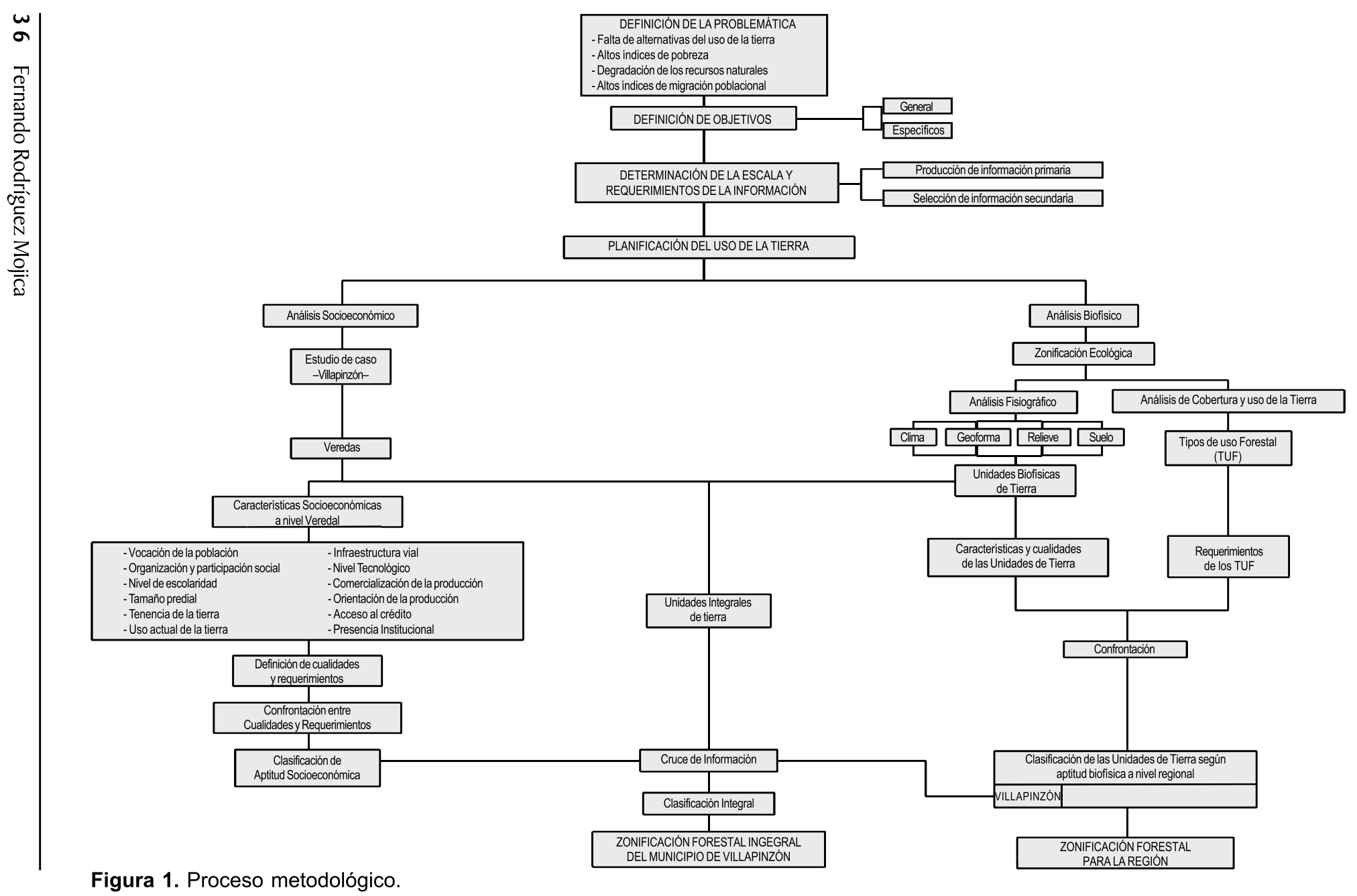


tudio "Metodología para delimitación de áreas homogéneas", realizado por el CIAF en 1981 en la cuenca del río Bogotá y presentado en un mapa de escala $1: 50.000$.

Con base en dicha información se definieron las variables: Piso altitudinal, Disponibilidad de agua, Condiciones de drenaje, Textura, Condiciones de enraizamiento, PH, y Resistencia a la erosión, a partir de las cuales se determinaron las cualidades y requerimientos, tal como aparecen en las tablas 1,2 y 4 .

Tabla 1. Requerimientos biofísicos del laurel de cera

\begin{tabular}{|c|c|c|c|c|c|c|c|}
\hline ALTITUD & $\begin{array}{c}\text { DISPONIBIL. } \\
\text { DE AGUA }\end{array}$ & $\begin{array}{c}\text { CONDICION } \\
\text { DE } \\
\text { DRENAJE }\end{array}$ & $\begin{array}{c}\text { CONDICIÓN } \\
\text { DE } \\
\text { ENRAIZAM. }\end{array}$ & PH & TEXTURA & $\begin{array}{c}\text { RESISTENCIA } \\
\text { A LA } \\
\text { EROSIÓN }\end{array}$ & $\begin{array}{c}\text { CLASE DE } \\
\text { APTITUD }\end{array}$ \\
\hline 3 & 2 & 1 & 1,2 & 1 & 2 & 3 & A1 \\
\hline 2 & 3 & 2 & 3 & 2 & 1,3 & 1,2 & A2 \\
\hline 1 & 1 & 3 & 4 & 3 & 4 & 4 & A3 \\
\hline$>3$ & 4 & 4 & $>4$ & 4,5 & 5 & $>4$ & $\mathrm{~N}$ \\
\hline
\end{tabular}

Fuente: Adaptado de Especies Vegetales Promisorias, 1998.

Tabla 2. Requerimientos biofísicos de la uva camarona

\begin{tabular}{|c|c|c|c|c|c|c|c|}
\hline ALTITUD & $\begin{array}{c}\text { DISPONIBIL. } \\
\text { DE AGUA }\end{array}$ & $\begin{array}{c}\text { CONDICION } \\
\text { DE } \\
\text { DRENAJE }\end{array}$ & $\begin{array}{c}\text { CONDICIÓN } \\
\text { DE } \\
\text { ENRAIZAM. }\end{array}$ & PH & TEXTURA & $\begin{array}{c}\text { RESISTENCIA } \\
\text { A LA } \\
\text { EROSIÓN }\end{array}$ & $\begin{array}{c}\text { CLASE DE } \\
\text { APTITUD }\end{array}$ \\
\hline 2 & 3 & 1 & 4,5 & 2,3 & 2 & 1,2 & A1 \\
\hline 1,3 & 2 & 2 & 2,3 & 1 & 1,3 & 3 & A2 \\
\hline$>3$ & 1 & 3 & 1 & 4 & 4 & 4 & A3 \\
\hline$>3$ & 4 & 4 & $<1$ & $>4$ & 5 & 4 & N \\
\hline
\end{tabular}

Fuente: Adaptado de Instituto de Ciencias Naturales, 1985.

Para establecer las cualidades de las unidades de tierra se hizo una categorización, indicando los diferentes grados que en términos cualitativos y/o cuantitativos presentan las características de las variables seleccionadas, lo cual originó una tabla síntesis, tabla 3, donde se muestra la situación real de cada unidad de tierra. 
Tabla 3. Cualidades codificadas de las unidades de tierra

\begin{tabular}{|c|c|c|c|c|c|c|c|}
\hline $\begin{array}{c}\text { CUALIDADES } \\
\text { DE LAS U.T. }\end{array}$ & ALTITUD & $\begin{array}{c}\text { DISPONIBIL. } \\
\text { DE } \\
\text { AGUA }\end{array}$ & $\begin{array}{c}\text { CONDICIÓN } \\
\text { DE } \\
\text { DRENAJE }\end{array}$ & $\begin{array}{c}\text { CONDICIÓN } \\
\text { DE } \\
\text { TEXTURA }\end{array}$ & ENRAIZ. & $\begin{array}{c}\text { RESISTENCIA } \\
\text { A LA } \\
\text { PH }\end{array}$ & $\begin{array}{c}\text { RESISTEN. } \\
\text { ALA } \\
\text { EROSIÓN }\end{array}$ \\
\hline SSC2 & 2 & 2 & 1 & 2 & 1 & 1 & 2 \\
\hline SSC3 & 2 & 2 & 1 & 2 & 2 & 1 & 3 \\
\hline SSG3 & 2 & 2 & 1 & 3 & 3 & 1 & 3 \\
\hline SUC2 & 2 & 1 & 1 & 5 & 2 & 1 & 2 \\
\hline SUC3 & 2 & 1 & 1 & 2 & 2 & 1 & 3 \\
\hline FSG2 & 3 & 2 & 1 & 2 & 1 & 1 & 2 \\
\hline FSG3 & 3 & 2 & 1 & 3 & 3 & 1 & 3 \\
\hline FST2 & 3 & 2 & 1 & 1 & 3 & 1 & 2 \\
\hline FSB2 & 3 & 2 & 1 & 3 & 1 & 1 & 2 \\
\hline FSB3 & 3 & 2 & 1 & 2 & 2 & 1 & 3 \\
\hline FSS1 & 3 & 2 & 1 & 3 & 1 & 1 & 1 \\
\hline FSS6 & 3 & 2 & 4 & 1 & 1 & 1 & 1 \\
\hline FSC5 & 3 & 2 & 1 & 2 & 4 & 1 & 3 \\
\hline FSE1 & 3 & 2 & 3 & 1 & 1 & 1 & 1 \\
\hline FUL6 & 3 & 1 & 4 & 1 & 2 & 2 & 1 \\
\hline FUG3 & 3 & 1 & 1 & 3 & 2 & 1 & 3 \\
\hline FEC5 & 3 & 4 & 1 & 2 & 4 & 4 & 2 \\
\hline FYC5 & 3 & 3 & 1 & 1 & 4 & 3 & 2 \\
\hline FYS1 & 3 & 3 & 2 & 1 & 3 & 4 & 1 \\
\hline FYG3 & 3 & 3 & 1 & 1 & 3 & 2 & 3 \\
\hline FYI1 & 3 & 3 & 4 & 1 & 1 & 1 & 1 \\
\hline
\end{tabular}

Finalmente, confrontando las tablas de cualidades y requerimientos, mediante una comparación individualizada de cada requerimiento con cada cualidad, se obtuvo la clasificación de la aptitud biofísica, la cual se presenta en un mapa donde se reagrupan las unidades de tierra que presentan el mismo grado de aptitud, determinando así la zonificación biofísica para cada una de las especies seleccionadas.
ANALISIS SOCIOECONOMICO: debido a la dificultad de poder contar con la información socioeconómica de cada una de las unidades de tierra, delimitadas mediante la integración de variables biofísicas, se consideraron las veredas como unidades espaciales para la realización de éste análisis.

Se definió una serie de cualidades teniendo en cuenta su pertinencia e im- 
Tabla 4. Características biofísicas de las unidades de tierra

\begin{tabular}{|c|c|c|c|c|c|c|c|c|}
\hline \begin{tabular}{|l} 
UNIDAD \\
DE \\
TIERRA
\end{tabular} & $\begin{array}{c}\text { PISO } \\
\text { ALTITUDINAL }\end{array}$ & $\begin{array}{l}\text { INDICEDE } \\
\text { HUMEDAD }\end{array}$ & $\begin{array}{l}\text { MATERIAL } \\
\text { LITOLOGICO }\end{array}$ & RELIEVE & $\begin{array}{l}\text { PROFUNDIDAD } \\
\text { EFECTIVA }\end{array}$ & TEXTURA & $\begin{array}{c}\text { CONDICION } \\
\text { DE } \\
\text { DRENAJE }\end{array}$ & $\mathrm{PH}$ \\
\hline SSC2 & Subpáramo(3000-3500m.s.n.m.) & Subhúmedo & Materiales complejos & Inclinado(12-25\%) & Suelos profundos & Franco-arcillosa & Bien drenados & Fuertemente ácido \\
\hline $\mathrm{ssc} 3$ & Subpáramo(3000-3500m.s.n.m.) & Subhúmedo & Materiales complejos & Moderadamente escarpado(25-75\%) & Suelos medianamente profundos & Franco-arcillosa & Bien drenados & Fuertemente ácido \\
\hline SSG3 & Subpáramo(3000-3500m.s.n.m.) & Subhúmedo & Arenisca cuarcitica & Moderadamente escarpado(25-75\%) & Suelos superficiales & Franca & Bien drenados & Fuertemente ácido \\
\hline SUC2 & Subpáramo(3000-3500m.s.n.m.) & Húmedo & Materiales complejos & Inclinado(12-25\%) & Suelos medianamente profundos & Arenosa & Bien drenados & Fuertemente ácido \\
\hline SUC3 & Subpáramo(3000-3500m.s.n.m.) & Húmedo & Materiales complejos & Moderadamente escarpado(25-75\%) & Suelos medianamente profundos & Franco-arcillosa & Bien drenados & Fuertemente ácido \\
\hline FSG2 & Frio(2000-3000m.s.n.m.) & Subhúmedo & Arenisca cuarcítica & Inclinado(12-25\%) & Suelos profundos & Franco-arcillosa & Bien drenados & Fuertemente ácido \\
\hline FSG3 & Frio(2000-3000m.s.n.m.) & Subhúmedo & Arenisca cuarcítica & Moderadamente escarpado(25-75\%) & Suelos superficiales & Franca & Bien drenados & Fuertemente ácido \\
\hline FST2 & Frio(2000-3000m.s.n.m.) & Subhúmedo & Ceniza volcánica s obre arena & Inclinado(12-25\%) & Suelos superficiales & Arcillosa & Bien drenados & Fuertemente ácido \\
\hline FSB2 & Frio(2000-3000m.s.n.m.) & Subhúmedo & Arcillolita & Inclinado(12-25\%) & Suelos profundos & Franca & Bien drenados & Fuertemente ácido \\
\hline FSB3 & Frio(2000-3000m.s.n.m.) & Subhúmedo & Arcillolita & Moderadamente escarpado(25-75\%) & Suelos medianamente profundos & Franco-arcillosa & Bien drenados & Fuertemente ácido \\
\hline FSS1 & Frio(2000-3000m.s.n.m.) & Subhúmedo & Ceniza volcánica sobre arcilla & Ligeramente inclinado (0-12\%) & Suelos profundos & Franca & Bien drenados & Fuertemente ácido \\
\hline FSS6 & Frio(2000-3000m.s.n.m.) & Subhúmedo & Ceniza volcánica sobre arcilla & Ligeramente inclinado (0-12\%) & Suelos profundos & Arcillosa & Drenaje imperfecto & Fuertemente ácido \\
\hline FSC5 & Frio(2000-3000m.s.n.m.) & Subhúmedo & Materiales complejos & Moderadamente escarpado(25-75\%) & Suelos muy superficiales & Franco-arcillosa & Bien drenados & Fuertemente ácido \\
\hline FSE1 & Frio(2000-3000m.s.n.m.) & Subhúmedo & Valles coluvio-aluviales & Ligeramente inclinado $(0-12 \%)$ & Suelos profundos & Arcillosa & Pobremente drenado & Fuertemente ácido \\
\hline FUL6 & Frio(2000-3000m.s.n.m.) & Húmedo & Llanura aluvial de inundación & Ligeramente inclinado (0-12\%) & Suelos medianamente profundos & Arcillosa & Drenaje imperfecto & Acido \\
\hline FUG3 & Frio(2000-3000m.s.n.m.) & Húmedo & Arenisca cuarcítica & Moderadamente escarpado(25-75\%) & Suelos medianamente profundos & Franca & Bien drenados & Fuertemente ácido \\
\hline FEC5 & Frio(2000-3000m.s.n.m.) & Semiárido & Materiales complejos & Inclinado(12-25\%) & Suelos muy superficiales & Franco-arcillosa & Bien drenados & Moderadam. alcalinc \\
\hline FYC5 & Frio(2000-3000m.s.n.m.) & Subhúmedo-seco & Materiales complejos & Inclinado(12-25\%) & Suelos muy superficiales & Arcillosa & Bien drenados & Neutro \\
\hline FYS1 & Frio(2000-3000m.s.n.m.) & Subhúmedo-seco & Ceniza volcánica sobre arcilla & Ligeramente inclinado $(0-12 \%)$ & Suelos superficiales & Arcillosa & Moderadam. drenado & Ligeramente alcalinc \\
\hline FYG3 & Frio(2000-3000m.s.n.m.) & Subhúmedo-seco & Arenisca cuarcítica & Moderadamente escarpado(25-75\%) & Suelos superficiales & Arcillosa & Bien drenados & Ligeramente ácido \\
\hline FYI1 & Frio(2000-3000m.s.n.m.) & Subhúmedo-seco & Aluviones sobre arcilla lacustre & Plano (0-3\%) & Suelos profundos & Arcillosa & Drenaje imperfecto & Fuertemente ácido \\
\hline
\end{tabular}

Fuente: CIAF. 1981 
portancia en la valoración de las necesidades de mejoramiento en las condiciones de vida de la población, y que a la vez complementaran los resultados obtenidos en el análisis biofísico, reafirmándolos o descartándolos, como información confiable para la toma de decisiones respecto a sus posibilidades de desarrollo en este territorio geográfico.

Con base en la información obtenida a partir de fuentes primarias, mediante entrevistas con funcionarios de las instituciones oficiales existentes en el municipio de Villapinzón y charlas informales con algunos pobladores del área rural, así como fuentes secundarias a través de revisión de documentos y estudios realizados y observaciones directas de campo, se seleccionaron las siguientes variables: Vocación de la población, Organización y participación social de la comunidad, Nivel de escolaridad, Tamaños prediales, Tenencia de la tierra, Uso actual de la tierra, Nivel tecnológico, Infraestructura vial, Comerciali-zación de los productos, Orientación de la producción, Acceso al crédito y Presencia institucional.

Se estableció una categorización para cada una de estas variables con el propósito de definir las tablas de cualidades y requerimientos, tablas 5 y 6 , a partir de las características de cada variable dentro de las veredas consideradas, información que posterior- mente se confrontó para así obtener la clasificación de la aptitud socioeconómica de las diferentes veredas, la cual se consignó en un mapa donde se agrupan las unidades espaciales que presentan el mismo grado de aptitud para la implementación de las especies forestales seleccionadas.

ANALISIS INTEGRAL: este análisis se llevó a cabo relacionando la clasificación final de aptitud de las formas biofísicas con la clasificación final de los aspectos socioeconómicos, para cada una de las unidades espaciales (unidades socioambientales) definidas mediante la superposición cartográfica de las unidades de tierra determinadas en términos biofísicos y los límites veredales, a las cuales se les asignó un nuevo código, compuesto por el originalmente establecido para las unidades de tierra más un subíndice que indica el código asignado a cada una de las 13 veredas localizadas dentro de la cuenca del río Bogotá.

El resultado de esta comparación determinó una clasificación integral de aptitud, presentada en un mapa donde se agrupan las unidades espaciales con el mismo grado de aptitud, obteniendo así la Zonificación Forestal Integral del municipio de Villapinzón, la cual representa una información más ajustada y objetiva respecto a las verdaderas posibilidades de implementación de las especies forestales Laurel de cera y Uva camarona. 
Tabla 5. Requerimientos socioeconómicos laurel de cera y uva camarona

\begin{tabular}{|l|c|c|c|c|}
\hline \multicolumn{1}{|c|}{ Variables } & a1 & $\mathbf{a 2}$ & $\mathbf{a 3}$ & $\mathbf{n}$ \\
\hline Vocacion de la población & 1 & 2 & 2.3 & 3 \\
\hline Organización y participacion social & 1 & 2 & 2.3 & 3 \\
\hline Nivel de escolaridad & 2 & 1 & 2.3 & 3 \\
\hline Tamaños prediales & 2 & 1 & 2.3 & 3 \\
\hline Tenencia de la tierra & 1 & 2 & 2.3 & 3 \\
\hline Uso actual de la tierra & 1 & 2 & 2.3 & 3 \\
\hline Nivel tecnológico & 1 & 2 & 2.3 & 3 \\
\hline Infraestructura vial & 1.2 & 3 & 3.4 & 4 \\
\hline Comercialización de la producción & 2 & 1 & 2.3 & 3 \\
\hline Orientación de la producción & 2 & 1 & 2.3 & 3 \\
\hline Acceso al crédito & 1 & 2 & 3 & 4 \\
\hline Presencia institucional & 1 & 2 & 2.3 & 3 \\
\hline
\end{tabular}

Fuente: Autor.

\section{Resultados}

Los resultados se organizan en dos componentes, el de la región y el del municipio de Villapinzón. El bloque de resultados de la región contiene información exclusiva sobre la aptitud biofísica de 21 unidades de tierra, mientras que el de Villapinzón contiene la aptitud integral para las 57 unidades socioambientales obtenidas del cruce de la información biofísica de 8 unidades de tierra y de la información socioeconómica de las 13 veredas.

Aptitud biofísica para laurel de cera: como se observa en la tabla 8 en la región se encuentran todas las clases de aptitud biofísica, destacándose la unidad de tierra identificada como $\mathrm{FSG}_{2}$ con un alto grado de aptitud A1, caracterizada por encontrarse en un clima frío, subhúmedo, con presencia de colinas de areniscas con un relieve inclinado, localizada en su mayor parte en el municipio de Suesca.

Las unidades de tierra que presentan grados de aptitud entre moderada (A2) y marginal (A3) deben este hecho principalmente a la presencia de limitantes de tipo edáfico como la textura del suelo, la resistencia a la erosión y la condición de enraizamiento, y de tipo climático como la altitud, mientras que aquellas clasificadas como no aptas $(\mathrm{N})$ presentan grandes limitaciones que en la actualidad im- 
Tabla 6. Cualidades socioeconómicas codificadas de las veredas del municipio de Villapinzon

\begin{tabular}{|c|c|c|c|c|c|c|c|c|c|c|c|c|}
\hline VEREDA & $\begin{array}{c}\text { VOCACIÓN } \\
\text { DE LA } \\
\text { POBLACIÓN }\end{array}$ & $\begin{array}{c}\text { ORGANIZ. Y } \\
\text { PARTICIP. } \\
\text { SOCIAL }\end{array}$ & $\begin{array}{c}\text { NIVEL } \\
\text { DE ESCO- } \\
\text { LARIDAD }\end{array}$ & $\begin{array}{c}\text { TAMAÑOS } \\
\text { PREDIA- } \\
\text { LES }\end{array}$ & $\begin{array}{c}\text { TENENCIA } \\
\text { DE LA } \\
\text { TIERRA }\end{array}$ & $\begin{array}{c}\text { USO } \\
\text { ACTUAL DE } \\
\text { LA TIERRA }\end{array}$ & $\begin{array}{c}\text { NIVEL } \\
\text { TECNO- } \\
\text { LÓGICO }\end{array}$ & $\begin{array}{c}\text { INFRAES- } \\
\text { ESTRUCT. } \\
\text { VIAL }\end{array}$ & $\begin{array}{c}\text { COMERC. } \\
\text { DE LA } \\
\text { PRODUC. }\end{array}$ & $\begin{array}{c}\text { ORIENT. } \\
\text { DE LA } \\
\text { PRODUC. }\end{array}$ & $\begin{array}{c}\text { ACCESO } \\
\text { AL } \\
\text { CRÉDITO }\end{array}$ & $\begin{array}{c}\text { PRESENCIA } \\
\text { INSTITU- } \\
\text { CIONAL }\end{array}$ \\
\hline BOSAVITA & 1 & 2 & 3 & 2 & 2 & 3 & 1 & 2 & 2 & 1 & 3 & 2 \\
\hline CASA BLANCA & 3 & 3 & 3 & 3 & 2 & 1 & 3 & 4 & 2 & 3 & 3 & 2 \\
\hline CHASQUEZ & 1 & 1 & 3 & 2 & 1 & 1 & 1 & 2 & 2 & 2 & 3 & 1 \\
\hline CHIGUALA & 2 & 3 & 3 & 3 & 2 & 1 & 3 & 2 & 2 & 2 & 3 & 2 \\
\hline GUANGUITA & 1 & 1 & 3 & 2 & 2 & 3 & 1 & 2 & 2 & 1 & 3 & 1 \\
\hline JOYA & 2 & 3 & 3 & 3 & 2 & 1 & 3 & 4 & 2 & 3 & 3 & 2 \\
\hline LA MERCED & 1 & 3 & 3 & 3 & 1 & 2 & 1 & 2 & 2 & 1 & 3 & 2 \\
\hline QUINCHA & 1 & 2 & 3 & 3 & 2 & 1 & 1 & 3 & 2 & 2 & 3 & 2 \\
\hline REATOVA & 2 & 2 & 3 & 3 & 2 & 1 & 3 & 3.4 & 2 & 3 & 3 & 2 \\
\hline SALITRE & 1 & 1 & 3 & 2 & 1 & 3 & 1 & 3.4 & 2 & 1 & 3 & 1 \\
\hline SAN PABLO & 1 & 3 & 3 & 2 & 1 & 3 & 2 & 3.4 & 2 & 1 & 3 & 2 \\
\hline SAN PEDRO & 3 & 3 & 3 & 3 & 2 & 1 & 3 & 3.4 & 2 & 3 & 3 & 2 \\
\hline SONSA & 1 & 1 & 3 & 3 & 2 & 3 & 1 & 3.4 & 2 & 2 & 3 & 1 \\
\hline
\end{tabular}

Fuente: Autor. 
piden toda posibilidad de lograr una producción sostenible, debido a deficientes condiciones de drenaje de los suelos y a sus altos niveles de concentración de hidrogeniones $(\mathrm{Ph})$.

En la tabla 7 se observa la superficie ocupada en cada municipio para los diferentes grados de aptitud, destacándose la presencia de una importante superficie con un grado de aptitud moderada (A2), localizada en el municipio de Chocontá, como se aprecia en la figura 2. Su representación espacial se presenta en el mapa 2.

Tabla 7. Superficie ocupada por grado de aptitud por municipio (Ha)

\begin{tabular}{|l|r|r|r|r|}
\hline \multirow{2}{*}{ MUNICIPIO } & \multicolumn{4}{|c|}{ GRADO DE APTITUD } \\
\cline { 2 - 5 } & $\mathbf{A 1}$ & $\mathbf{A 2}$ & $\mathbf{A 3}$ & $\mathbf{N}$ \\
\hline CHOCONTA & $1.384,52$ & $20.383,31$ & $2.137,42$ & $1.324,31$ \\
\hline COGUA & 0 & $10.377,96$ & 911,40 & $1.574,06$ \\
\hline GUASCA & 0 & $6.293,82$ & $9.383,24$ & $5.650,53$ \\
\hline GUATAVITA & 0 & $6.498,82$ & $3.581,06$ & $2.773,30$ \\
\hline NEMOCON & 4,35 & $3.124,53$ & 0,09 & $6.696,05$ \\
\hline SESQUILE & 778,33 & $7.593,65$ & $2.687,64$ & $2.163,93$ \\
\hline SUESCA & $2.171,21$ & $1.609,65$ & 518,67 & $6.143,03$ \\
\hline VILLAPINZÓN & $1.610,60$ & $10.550,94$ & 514,69 & 229,61 \\
\hline TOTAL & $\mathbf{5 . 9 4 9 , 0 1}$ & $\mathbf{6 6 . 4 3 2 , 6 8}$ & $\mathbf{1 9 . 7 3 4 , 2 1}$ & $\mathbf{2 6 . 5 5 4 , 8 2}$ \\
\hline
\end{tabular}

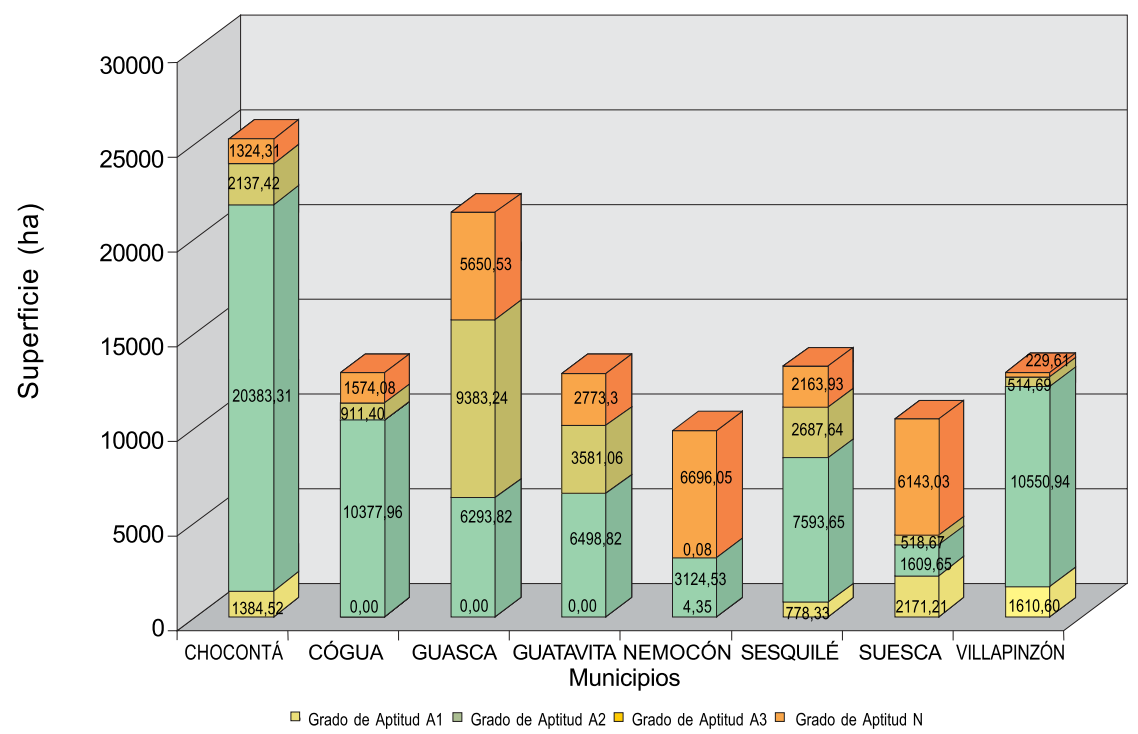

Figura 2. Superficie ocupada por grado de aptitud por municipio para Laurel de Cera. 
Tabla 8. Clasificacion de la aptitud biofisica para laurel de cera

\begin{tabular}{|l|c|c|c|c|c|c|c|c|}
\hline $\begin{array}{c}\text { UNIDAD } \\
\text { DE } \\
\text { TIERRA }\end{array}$ & ALTITD & $\begin{array}{c}\text { DISPONIB. } \\
\text { DE } \\
\text { AGUA }\end{array}$ & $\begin{array}{c}\text { CONDICIÓN } \\
\text { DE } \\
\text { DRENAJE }\end{array}$ & TEXTURA & $\begin{array}{c}\text { CONDICIÓN } \\
\text { DE } \\
\text { ENRAZAM. }\end{array}$ & PH & $\begin{array}{c}\text { RESISTENCIA } \\
\text { ALA } \\
\text { EROSIÓN }\end{array}$ & $\begin{array}{c}\text { CLASE } \\
\text { DE } \\
\text { APTITUD }\end{array}$ \\
\hline SSC2 & A2 & A1 & A1 & A1 & A1 & A1 & A1 & A2 \\
\hline SSC3 & A2 & A1 & A1 & A1 & A1 & A1 & A2 & A2 \\
\hline SSG3 & A2 & A1 & A1 & A2 & A2 & A1 & A2 & A2 \\
\hline SUC2 & A2 & A3 & A1 & N & A1 & A1 & A1 & N \\
\hline SUC3 & A2 & A3 & A1 & A1 & A1 & A1 & A2 & A3 \\
\hline FSG2 & A1 & A1 & A1 & A1 & A1 & A1 & A1 & A1 \\
\hline FSG3 & A1 & A1 & A1 & A2 & A2 & A1 & A2 & A2 \\
\hline FST2 & A1 & A1 & A1 & A2 & A2 & A1 & A1 & A2 \\
\hline FSB2 & A1 & A1 & A1 & A2 & A1 & A1 & A1 & A2 \\
\hline FSB3 & A1 & A1 & A1 & A1 & A1 & A1 & A2 & A2 \\
\hline FSS1 & A1 & A1 & A1 & A2 & A1 & A1 & A1 & A2 \\
\hline FSS6 & A1 & A1 & N & A2 & A1 & A1 & A1 & N \\
\hline FSC5 & A1 & A1 & A1 & A1 & A3 & A1 & A2 & A3 \\
\hline FSE1 & A1 & A1 & A3 & A2 & A1 & A1 & A1 & A3 \\
\hline FUL6 & A1 & A3 & N & A2 & A1 & A2 & A1 & N \\
\hline FUG3 & A1 & A3 & A1 & A2 & A1 & A1 & A2 & A3 \\
\hline FEC5 & A1 & N & A1 & A1 & A3 & N & A1 & N \\
\hline FYC5 & A1 & A2 & A1 & A2 & A3 & A1 & A3 \\
\hline FYS1 & A1 & A2 & A2 & A2 & A2 & N & A1 & N \\
\hline FYG3 & A1 & A2 & A1 & A2 & A2 & A2 & A2 & A2 \\
\hline FYI1 & A1 & A2 & N & A2 & A1 & A1 & A1 & N \\
\hline
\end{tabular}

\section{Aptitud biofísica para uva camarona:}

De la tabla 10 se puede observar que dentro de la región no existen áreas de alta aptitud (A1) para la implementación de esta especie, mientras que una gran superficie es de aptitud marginal (A3) y nula $(\mathrm{N})$ debido principalmente a problemas relaciona- dos con la textura, disponibilidad de agua y condiciones de drenaje; las áreas con aptitud moderada (A2) representan una considerable extensión con limitaciones originadas por la altitud $\mathrm{Ph}, \mathrm{y}$ disponibilidad de agua. Su distribución por municipio se presenta en la tabla 9 y en la figura $3 \mathrm{y}$ espacialmente en el mapa 3. 
Tabla 9. Superficie ocupada por grado de aptitud por municipio (Ha)

\begin{tabular}{|l|c|r|r|r|}
\hline & \multicolumn{4}{|c|}{ GRADO DE APTITUD } \\
\hline MUNICIPIO & $\mathbf{A 1}$ & \multicolumn{1}{|c|}{$\mathbf{A 2}$} & \multicolumn{1}{c|}{$\mathbf{A 3}$} & \multicolumn{1}{c|}{ N } \\
\hline CHOCONTA & 0 & $8.521,76$ & $8.282,41$ & $8.425,39$ \\
\hline COGUA & 0 & $8.728,00$ & $3.282,95$ & 852,47 \\
\hline GUASCA & 0 & $6.291,91$ & $7.628,75$ & $7.406,93$ \\
\hline GUATAVITA & 0 & $5.437,70$ & $2.207,45$ & $5.208,03$ \\
\hline NEMOCON & 0 & 880,75 & 4,35 & $8.939,92$ \\
\hline SESQUILE & 0 & $7.592,06$ & $2.473,38$ & $3.158,11$ \\
\hline SUESCA & 0 & 453,82 & $2.686,16$ & $7.302,58$ \\
\hline VILLAPINZÓN & 0 & $3.289,05$ & $7.141,02$ & $2.475,77$ \\
\hline TOTAL & $\mathbf{0}$ & $\mathbf{4 1 . 1 9 5 , 1 2}$ & $\mathbf{3 3 . 7 0 6 , 4 8}$ & $\mathbf{4 3 . 7 6 9 , 2 0}$ \\
\hline
\end{tabular}

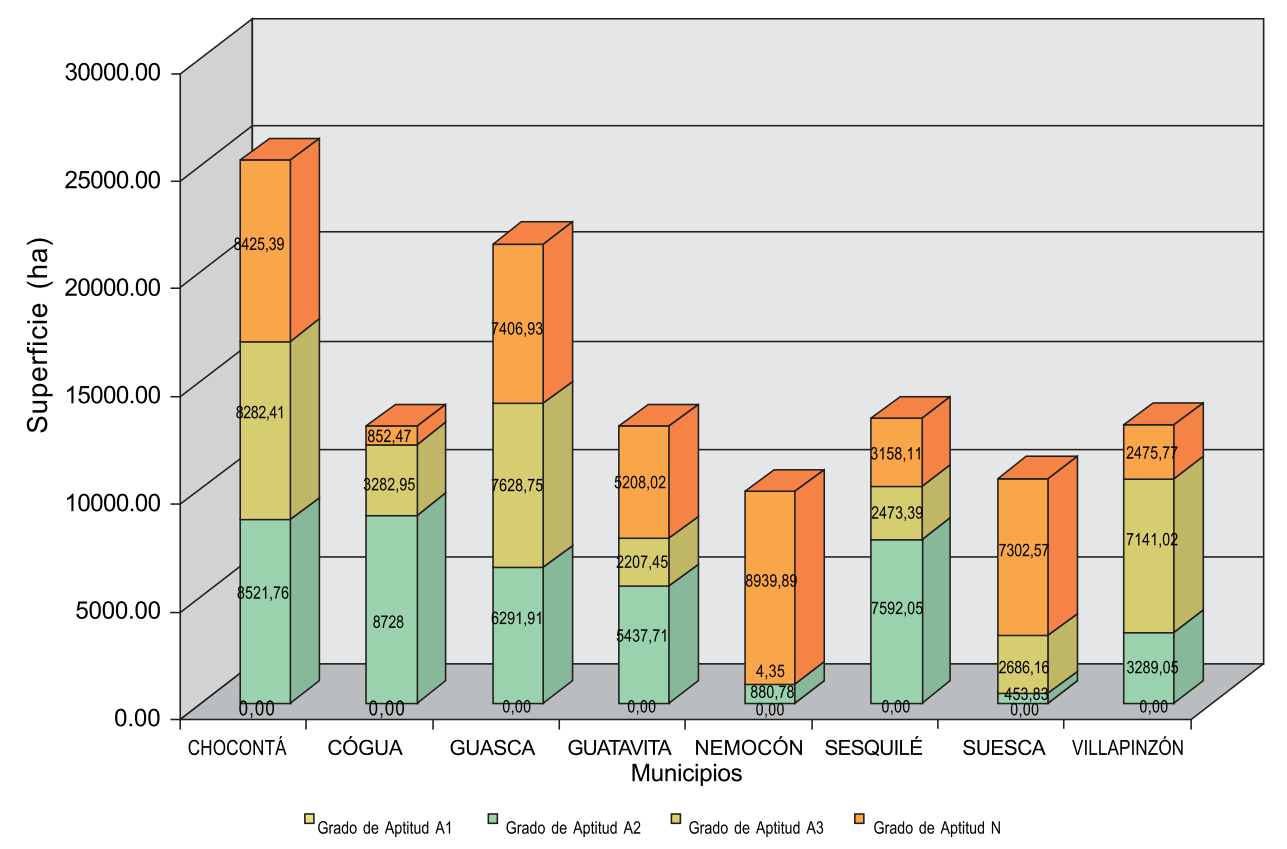

Figura 3. Superficie ocupada por grado de aptitud por municipio para Uva Camarona. 
Tabla 10. Clasificacion de la aptitud biofisica para Uva Camarona

\begin{tabular}{|c|c|c|c|c|c|c|c|c|}
\hline $\begin{array}{c}\text { UNIDAD } \\
\text { DE } \\
\text { TIERRA }\end{array}$ & ALTITUD & $\begin{array}{c}\text { DISPONIBILIDAD } \\
\text { DE } \\
\text { AGUA }\end{array}$ & $\begin{array}{c}\text { CONDICION } \\
\text { DE } \\
\text { DRENAJE }\end{array}$ & TEXTURA & $\begin{array}{c}\text { CONDICION } \\
\text { DE } \\
\text { ENRAIZAMIENTO }\end{array}$ & PH & $\begin{array}{c}\text { RESISTENCIA } \\
\text { A LA } \\
\text { EROSION }\end{array}$ & $\begin{array}{l}\text { CLASE } \\
\text { DE } \\
\text { APTITUD }\end{array}$ \\
\hline $\mathrm{SSC} 2$ & A1 & $\mathrm{A} 2$ & A1 & A3 & A1 & $\mathrm{A} 2$ & A1 & A3 \\
\hline SSC3 & $\mathrm{A} 1$ & $\mathrm{~A} 2$ & $\mathrm{~A} 1$ & A3 & $\mathrm{A} 1$ & $\mathrm{~A} 2$ & $\mathrm{~A} 2$ & $\mathbf{A 3}$ \\
\hline SSG3 & A1 & $\mathrm{A} 2$ & A1 & $\mathrm{A} 2$ & $\mathrm{~A} 2$ & $\mathrm{~A} 2$ & $\mathrm{~A} 2$ & A2 \\
\hline SUC2 & A1 & A3 & A1 & A1 & A1 & $\mathrm{A} 2$ & A1 & A3 \\
\hline SUC3 & A1 & A3 & A1 & A3 & A1 & $\mathrm{A} 2$ & A2 & A3 \\
\hline FSG2 & $\mathrm{A} 2$ & $\mathrm{~A} 2$ & $\mathrm{~A} 1$ & A3 & $\mathrm{A} 1$ & $\mathrm{~A} 2$ & A1 & $\mathbf{A 3}$ \\
\hline FSG3 & $\mathrm{A} 2$ & $\mathrm{~A} 2$ & A1 & $\mathrm{A} 2$ & $\mathrm{~A} 2$ & $\mathrm{~A} 2$ & $\mathrm{~A} 2$ & A2 \\
\hline FST2 & $\mathrm{A} 2$ & $\mathrm{~A} 2$ & $\mathrm{~A} 1$ & $\mathrm{~N}$ & $\mathrm{~A} 2$ & $\mathrm{~A} 2$ & $\mathrm{~A} 1$ & $\mathbf{N}$ \\
\hline FSB2 & $\mathrm{A} 2$ & $\mathrm{~A} 2$ & A1 & $\mathrm{A} 2$ & A1 & $\mathrm{A} 2$ & $\mathrm{~A} 1$ & A2 \\
\hline FSB3 & $\mathrm{A} 2$ & $\mathrm{~A} 2$ & $\mathrm{~A} 1$ & A3 & A1 & $\mathrm{A} 2$ & $\mathrm{~A} 2$ & A3 \\
\hline FSS1 & $\mathrm{A} 2$ & $\mathrm{~A} 2$ & A1 & $\mathrm{A} 2$ & A1 & $\mathrm{A} 2$ & A1 & A2 \\
\hline FSS6 & $\mathrm{A} 2$ & $\mathrm{~A} 2$ & $\mathrm{~N}$ & $\mathrm{~N}$ & A1 & $\mathrm{A} 2$ & $\mathrm{~A} 1$ & $\mathbf{N}$ \\
\hline FSC5 & $\mathrm{A} 2$ & $\mathrm{~A} 2$ & A1 & A3 & $\mathrm{A} 2$ & $\mathrm{~A} 2$ & $\mathrm{~A} 2$ & A3 \\
\hline FSE1 & $\mathrm{A} 2$ & $\mathrm{~A} 2$ & A3 & $\mathrm{N}$ & A1 & $\mathrm{A} 2$ & $\mathrm{~A} 1$ & $\mathbf{N}$ \\
\hline FUL6 & $\mathrm{A} 2$ & $\mathrm{~A} 3$ & $\mathrm{~N}$ & $\mathrm{~N}$ & $\mathrm{~A} 1$ & $\mathrm{~A} 1$ & $\mathrm{~A} 1$ & $\mathbf{N}$ \\
\hline FUG3 & $\mathrm{A} 2$ & A3 & $\mathrm{A} 1$ & $\mathrm{~A} 2$ & A1 & $\mathrm{A} 2$ & $\mathrm{~A} 2$ & A3 \\
\hline FEC5 & $\mathrm{A} 2$ & $\mathrm{~N}$ & $\mathrm{~A} 1$ & A3 & $\mathrm{A} 2$ & A3 & $\mathrm{A} 1$ & $\mathbf{N}$ \\
\hline FYC5 & $\mathrm{A} 2$ & A1 & A1 & $\mathrm{N}$ & $\mathrm{A} 2$ & $\mathrm{~A} 2$ & $\mathrm{~A} 1$ & $\mathbf{N}$ \\
\hline FYS1 & $\mathrm{A} 2$ & A1 & $\mathrm{A} 2$ & $\mathrm{~N}$ & $\mathrm{~A} 2$ & A3 & $\mathrm{A} 1$ & $\mathbf{N}$ \\
\hline FYG3 & $\mathrm{A} 2$ & $\mathrm{~A} 1$ & $\mathrm{~A} 1$ & $\mathrm{~N}$ & $\mathrm{~A} 2$ & $\mathrm{~A} 1$ & $\mathrm{~A} 2$ & $\mathbf{N}$ \\
\hline FYI1 & $\mathrm{A} 2$ & A1 & $\mathrm{N}$ & $\mathrm{N}$ & $\mathrm{A} 2$ & $\mathrm{~A} 2$ & $\mathrm{~A} 1$ & $\mathbf{N}$ \\
\hline
\end{tabular}




\section{Aptitud integral del municipio de Villapinzón}

Los resultados se organizaron en tres componentes, aptitud biofísica de las unidades de tierra, aptitud socioeconómica de las veredas y aptitud integral de las unidades socioambientales; para cada uno de estos componentes se utilizan unidades de análisis diferentes con el objeto de garantizar el mayor grado de desagregación y comprensión de la información aquí contenida.

\section{Aptitud biofísica}

Aptitud biofísica para Laurel de cera: A partir de la tabla 11 se puede observar que en este municipio se presentan los diferentes grados de aptitud con predominio de una aptitud moderada (A2), cubriendo un área del $81.5 \%$ de la superficie del municipio, con una significativa incidencia de la textura como principal limitante de esta situación.

El $12.5 \%$ de la superficie representada por la unidad de tierra $\mathrm{FSG}_{2}$ ofrece las condiciones óptimas, alta aptitud (A1), para la implementación de esta especie, mientras que las zonas no aptas $(\mathrm{N})$ y con aptitud marginal (A3) ocupan sólo el $6 \%$ del total del municipio limitadas principalmente por las deficientes condiciones de enraizamiento y de drenaje, particularmente por su localización dentro de la llanura aluvial del río Bogotá, donde las continuas inundaciones afectan negativamente sus posibilidades de desarrollo, información que se puede observar desagregada a nivel de vereda en la figura 4.

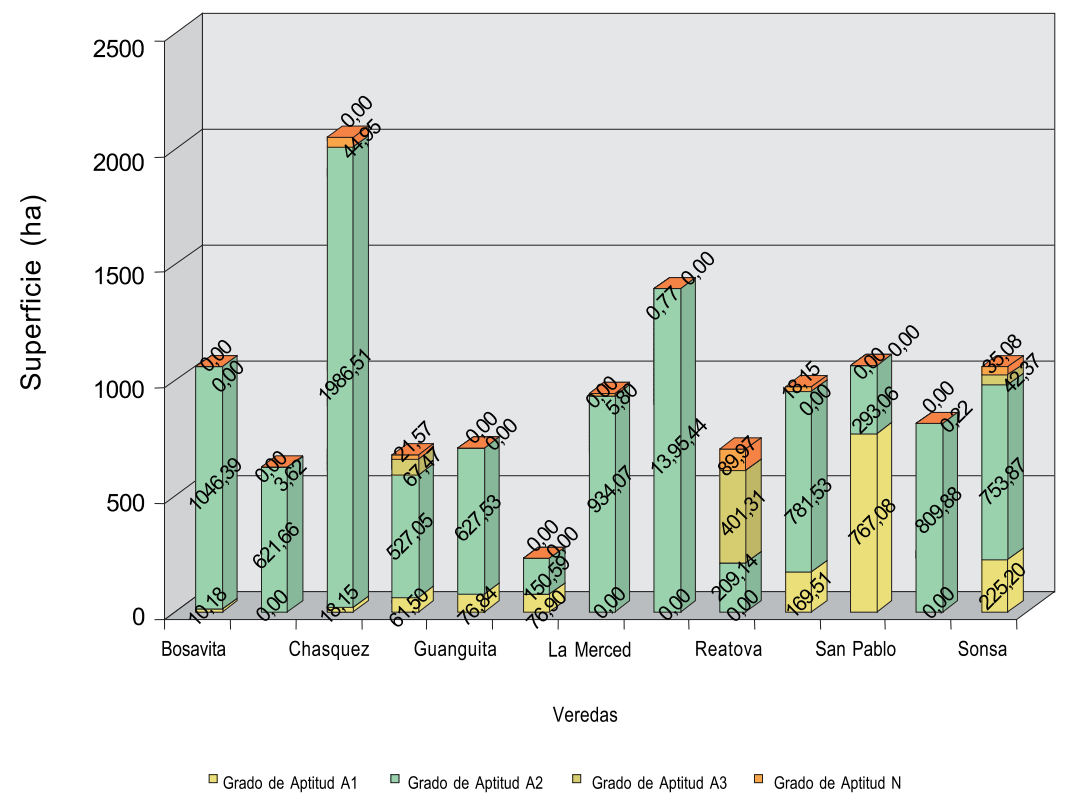

Figura 4. Superficie ocupada por grado de aptitud biofísica por vereda para Laurel de Cera en el municipio de Villapinzón. 
Tabla 11. Clasificación de la aptitud biofisica para Laurel de Cera en el municipio de Villapinzón

\begin{tabular}{|l|c|c|c|c|c|c|c|c|}
\hline $\begin{array}{c}\text { UNIDAD } \\
\text { DE } \\
\text { TIERRA }\end{array}$ & ALTITUD & $\begin{array}{c}\text { DISPONIBIL. } \\
\text { DE } \\
\text { AGUA }\end{array}$ & $\begin{array}{c}\text { CONDICION } \\
\text { DE } \\
\text { DRENAJ }\end{array}$ & TEXTURA & $\begin{array}{c}\text { CONDICION } \\
\text { DE } \\
\text { ENRAIZAM. }\end{array}$ & PH & $\begin{array}{c}\text { RESISTENCIA } \\
\text { A LA } \\
\text { EROSION }\end{array}$ & $\begin{array}{c}\text { CLASE } \\
\text { DE } \\
\text { APTITUD }\end{array}$ \\
\hline SSC3 & A2 & A1 & A1 & A1 & A1 & A1 & A2 & A2 \\
\hline FSG2 & A1 & A1 & A1 & A1 & A1 & A1 & A1 & A1 \\
\hline FSG3 & A1 & A1 & A1 & A2 & A2 & A1 & A2 & A2 \\
\hline FST2 & A1 & A1 & A1 & A2 & A2 & A1 & A1 & A2 \\
\hline FSB2 & A1 & A1 & A1 & A2 & A1 & A1 & A1 & A2 \\
\hline FSS1 & A1 & A1 & A1 & A2 & A1 & A1 & A1 & A2 \\
\hline FSC5 & A1 & A1 & A1 & A1 & A3 & A1 & A2 & A3 \\
\hline FUL6 & A1 & A3 & N & A2 & A1 & A2 & A1 & N \\
\hline
\end{tabular}

Aptitud biofísica para uva camarona: la clasificación final de la aptitud biofísica correspondiente a la uva camarona se muestra en la tabla 12 , donde se puede destacar la ausencia total de condiciones óptimas para su implementación, mientras que se observa que la mayor parte de las unidades de tierra presentan una aptitud entre nula $(\mathrm{N})$ y marginal (A3) con un $75 \%$ de la superficie del municipio, debido a limitaciones relacionadas con la textura de los suelos presentes en dichas unidades. El $25 \%$ restante corresponde a una aptitud moderada (A2), debido a problemas asociados al $\mathrm{Ph}$ de los suelos, información desagregada en la figura 5 .

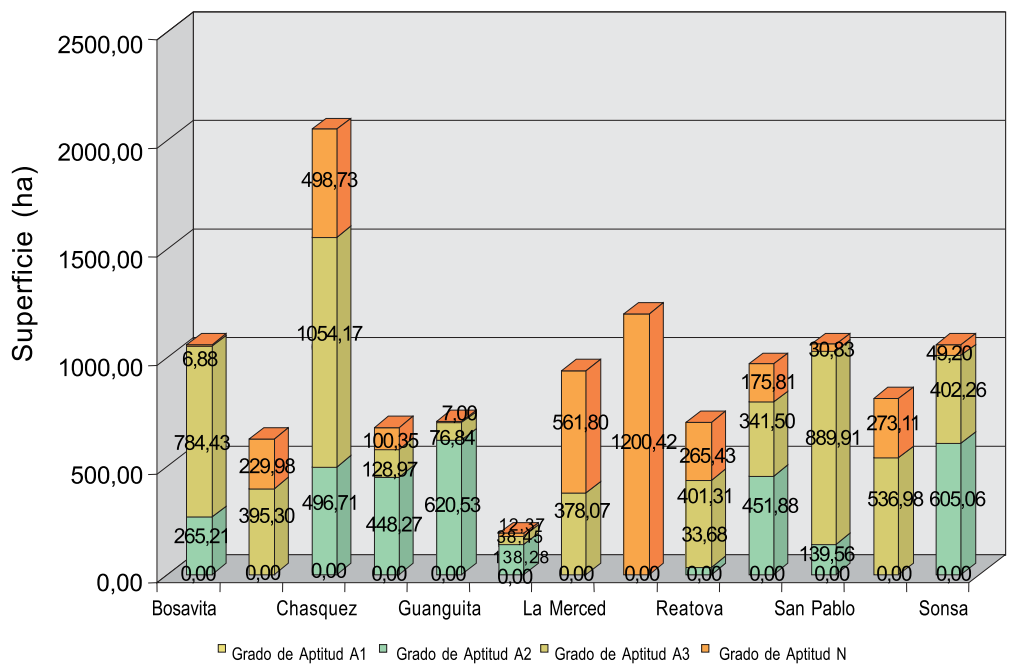

Figura 5. Superficie ocupada por grado de aptitud biofísica por vereda para Uva Camarona en el municipio de Villapinzón.

48 Fernando Rodríguez Mojica 
Tabla 12. Clasificación de la aptitud biofisica para Uva Camarona en el municipio de Villapinzón

\begin{tabular}{|l|c|c|c|c|c|c|c|c|}
\hline $\begin{array}{c}\text { UNIDAD } \\
\text { DE } \\
\text { TIERRA }\end{array}$ & ALTITUD & $\begin{array}{c}\text { DISPONIBIL. } \\
\text { DE } \\
\text { AGUA }\end{array}$ & $\begin{array}{c}\text { CONDICION } \\
\text { DE } \\
\text { DRENAJE }\end{array}$ & TEXTURA & $\begin{array}{c}\text { CONDICION } \\
\text { DE } \\
\text { ENRAIZAM. }\end{array}$ & PH & $\begin{array}{c}\text { RESISTENCIA } \\
\text { A LA } \\
\text { EROSION }\end{array}$ & $\begin{array}{c}\text { CLASE } \\
\text { DE } \\
\text { APTITUD }\end{array}$ \\
\hline SSC3 & A1 & A2 & A1 & A3 & A1 & A2 & A2 & A3 \\
\hline FSG2 & A2 & A2 & A1 & A3 & A1 & A2 & A1 & A3 \\
\hline FSG3 & A2 & A2 & A1 & A2 & A2 & A2 & A2 & A2 \\
\hline FST2 & A2 & A2 & A1 & N & A2 & A2 & A1 & N \\
\hline FSB2 & A2 & A2 & A1 & A2 & A1 & A2 & A1 & A2 \\
\hline FSS1 & A2 & A2 & A1 & A2 & A1 & A2 & A1 & A2 \\
\hline FSC5 & A2 & A2 & A1 & A3 & A2 & A2 & A3 & A3 \\
\hline FUL6 & A2 & A3 & N & N & A1 & A1 & A1 & N \\
\hline
\end{tabular}

\section{Aptitud socioeconómica}

Teniendo en cuenta que los aspectos socioeconómicos correspondientes a las cualidades de las veredas consideradas y a los requerimientos de las especies son exactamente los mismos, la confrontación entre estos originó una clasificación de aptitud socioeconómica idéntica para el Laurel de cera y para la Uva camarona, como se observa en la tabla 13.

Dicha información muestra que la totalidad de las veredas presentan un grado de aptitud marginal (A3), debido principalmente a la existencia de bajos niveles de escolaridad y a la poca cultura del uso de crédito formal, situación que se podría revertir con relativa facilidad mediante la acción decidida de los actores directamente relacionados con estas actividades, para lo cual se requiere establecer proce- sos de gestión más eficaces e integrados con los diferentes proyectos de desarrollo del municipio.

Los bajos niveles de escolaridad que actualmente presenta el municipio particularmente en sus áreas rurales, se debe principalmente a la falta de cobertura a nivel de educación secundaria, lo cual conduce a que los jóvenes una vez terminan su formación primaria, deben desplazarse a los centros urbanos con el fin de continuar sus estudios, incorporándose posteriormente a otras actividades productivas diferentes a las agropecuarias.

Además, no todas las personas que permanecen en el municipio logran terminar sus estudios primarios lo que aumenta la problemática relacionada con esta variable, desconociendo así el efecto positivo de la escuela en la modificación o afianzamiento de es- 
Tabla 13. Clasificacion de aptitud socioeconómica para laurel de cera

\begin{tabular}{|c|c|c|c|c|c|c|c|c|c|c|c|c|c|}
\hline VEREDA & $\begin{array}{l}\text { VOCACION } \\
\text { DE LA } \\
\text { POBLACIÓN }\end{array}$ & $\begin{array}{l}\text { ORGANIZAC.y } \\
\text { PARTICIPACIOON } \\
\text { SOCIAL }\end{array}$ & $\begin{array}{c}\text { NIVEL } \\
\text { DE } \\
\text { ESCOLARID. }\end{array}$ & $\begin{array}{l}\text { TAMAÑOS } \\
\text { PREDIALES }\end{array}$ & $\begin{array}{c}\text { TENENCIA } \\
\text { DE LA } \\
\text { TIERRA }\end{array}$ & $\begin{array}{c}\text { USO ACTUAL } \\
\text { DE LA } \\
\text { LA TIERRA }\end{array}$ & $\begin{array}{l}\text { NIVEL } \\
\text { TECNO- } \\
\text { LÓGICO }\end{array}$ & $\begin{array}{l}\text { INFRAES- } \\
\text { TRUCTURA } \\
\text { VIAL }\end{array}$ & $\begin{array}{l}\text { COMERCIAL. } \\
\text { DE LA } \\
\text { PRODUCCIÓN }\end{array}$ & $\begin{array}{c}\text { ORIENTAC. } \\
\text { DE LA } \\
\text { PRODUCCIÓN }\end{array}$ & $\begin{array}{l}\text { ACCESO } \\
\text { AL } \\
\text { CREDITO }\end{array}$ & $\begin{array}{l}\text { PRESENCIA } \\
\text { INSTITU- } \\
\text { CIONAL }\end{array}$ & $\begin{array}{l}\text { CLASE } \\
\text { DE } \\
\text { APTITUD }\end{array}$ \\
\hline BOSAVITA & A1 & $\mathrm{A} 2$ & A3 & A1 & $\mathrm{A} 2$ & A3 & A1 & A1 & A1 & $\mathrm{A} 2$ & A3 & $\mathrm{A} 2$ & A3 \\
\hline CASA BLANCA & A3 & A3 & A3 & A3 & $\mathrm{A} 2$ & A1 & A3 & A3 & A1 & A3 & A3 & $\mathrm{A} 2$ & A3 \\
\hline CHASQUEZ & $\mathrm{A} 1$ & A1 & A3 & A1 & A1 & $\mathrm{A} 1$ & A1 & A1 & $\mathrm{A} 1$ & A1 & A3 & A1 & A3 \\
\hline CHIGUALA & $\mathrm{A} 2$ & A3 & A3 & A3 & $\mathrm{A} 2$ & A1 & A3 & A1 & A1 & A1 & A3 & $\mathrm{A} 2$ & A3 \\
\hline GUANGUITA & $\mathrm{A} 1$ & $\mathrm{~A} 1$ & A3 & $\mathrm{A} 1$ & $\mathrm{~A} 2$ & A3 & A1 & $\mathrm{A} 1$ & $\mathrm{~A} 1$ & $\mathrm{~A} 2$ & A3 & A1 & A3 \\
\hline JOYA & $\mathrm{A} 2$ & A3 & A3 & A3 & $\mathrm{A} 2$ & $\mathrm{~A} 1$ & A3 & A3 & A1 & A3 & A3 & $\mathrm{A} 2$ & A3 \\
\hline LA MERCED & $\mathrm{A} 1$ & A3 & A3 & A3 & A1 & $\mathrm{A} 2$ & A1 & A1 & $\mathrm{A} 1$ & $\mathrm{~A} 2$ & A3 & $\mathrm{A} 2$ & A3 \\
\hline QUINCHA & A1 & $\mathrm{A} 2$ & A3 & A3 & $\mathrm{A} 2$ & A1 & A1 & $\mathrm{A} 2$ & A1 & A1 & A3 & $\mathrm{A} 2$ & A3 \\
\hline REATOVA & $\mathrm{A} 2$ & $\mathrm{~A} 2$ & A3 & A3 & $\mathrm{A} 2$ & A1 & A3 & A3 & A1 & A3 & A3 & $\mathrm{A} 2$ & A3 \\
\hline SALITRE & A1 & A1 & A3 & A1 & A1 & A3 & A1 & A3 & A1 & $\mathrm{A} 2$ & A3 & A1 & A3 \\
\hline SAN PABLO & A1 & A3 & A3 & A1 & A1 & A3 & $\mathrm{A} 2$ & A3 & A1 & $\mathrm{A} 2$ & A3 & $\mathrm{A} 2$ & A3 \\
\hline SAN PEDRO & A3 & A3 & A3 & A3 & $\mathrm{A} 2$ & A1 & A3 & A3 & A1 & A3 & A3 & $\mathrm{A} 2$ & A3 \\
\hline SONSA & A1 & $\mathrm{A} 1$ & A3 & A3 & $\mathrm{A} 2$ & A3 & A1 & A3 & $\mathrm{A} 1$ & A1 & A3 & $\mathrm{A} 1$ & A3 \\
\hline
\end{tabular}


quemas culturales tradicionales que permitan establecer una buena disponibilidad al cambio propuesto.

Los problemas relacionados con el acceso al crédito formal no se originan en la inexistencia de entidades crediticias, sino en la exigencia de un número de requisitos que hacen engorroso el proceso de desembolso, razón por lo que los habitantes acuden a esquemas de crédito informal que aunque más costosos, son más ágiles.

Aunque con un menor impacto, la variable correspondiente al uso actual de la tierra se convierte en una limitante a considerar sobre todo en aquellas veredas que presentan una significativa porción de su superficie bajo cultivo de papa, actividad arraigada a la cultura agropecuaria de la población, lo que haría difícil que en la actualidad se acepte un cambio radical en el uso de la tierra, situación que puede ser manejada con una buena campaña de educación ambiental, destacando las bondades de la actividad forestal, como garante de la sostenibilidad en la producción agropecuaria, principalmente en la producción del recurso hídrico.

\section{Aptitud integral}

La confrontación de la clasificación de la aptitud biofísica y de la clasificación socioeconómica produjo la clasificación de la aptitud integral de las unidades socioambientales como se ob- serva en la tabla 14, donde la mayor parte de estas presentan una aptitud integral marginal (A3) originada por limitantes de tipo socioeconómico, y en una menor proporción con una aptitud nula (N) producto de la presencia de limitantes de tipo biofísico.

Para el caso del Laurel de cera las unidades socioambientales que presentan un nulo grado de aptitud integral $(\mathrm{N})$, deben esta situación a problemas asociados a las características biofísicas de las unidades de tierra y en especial a las malas condiciones de drenaje, particularmente en la unidad de tierra $\mathrm{FLU}_{6}$, localizada en la mayor parte de las veredas que hacen parte de la llanura aluvial del río Bogotá, como se observa en el mapa 4.

En relación con la Uva camarona, las unidades con grado de aptitud integral nulo $(\mathrm{N})$ están asociadas con problemas de textura de los suelos, particularmente en la unidad de tierra $\mathrm{FST}_{2}$, así como también por las malas condiciones de drenaje en la unidad de tierra $\mathrm{FUL}_{6}$, dada su localización en la llanura aluvial del río Bogotá, como se observa en el mapa 5.

Las unidades socioambientales con aptitud integral marginal (A3), tanto para el Laurel de cera como para la Uva camarona, están condicionadas a limitaciones de tipo socioeconómico como consecuencia de los problemas relacionados con el nivel de escolaridad, el acceso al crédito y en algunos casos al uso de la tierra. 
Tabla 14. Aptitud integral municipio de Villapinzón

\begin{tabular}{|c|c|c|c|c|c|c|}
\hline \multicolumn{3}{|c|}{ APTITUD INTEGRAL LAUREL DE CERA } & \multirow{2}{*}{$\begin{array}{c}\text { UNIDADES } \\
\text { SOCIO } \\
\text { AMBIENTALES }\end{array}$} & \multicolumn{3}{|c|}{ APTITUD INTEGRAL UVA CAMARONA } \\
\hline $\begin{array}{c}\text { Aptitud } \\
\text { Biofisica }\end{array}$ & $\begin{array}{c}\text { Aptitud } \\
\text { Socioeconómica }\end{array}$ & $\begin{array}{l}\text { Aptitud } \\
\text { Integral }\end{array}$ & & $\begin{array}{c}\text { Aptitud } \\
\text { Biofisica }\end{array}$ & $\begin{array}{c}\text { Aptitud } \\
\text { Socioeconómica }\end{array}$ & $\begin{array}{l}\text { Aptitud } \\
\text { Integral }\end{array}$ \\
\hline $\mathrm{A} 2$ & $\mathrm{~A} 3$ & $\mathbf{A 3}$ & SSC31 & $\mathrm{A} 3$ & $\mathrm{~A} 3$ & $\mathbf{A 3}$ \\
\hline $\mathrm{A} 2$ & $\mathrm{~A} 3$ & A3 & FSB21 & $\mathrm{A} 2$ & $\mathrm{~A} 3$ & $\mathbf{A 3}$ \\
\hline $\mathrm{A} 1$ & $\mathrm{~A} 3$ & $\mathbf{A 3}$ & FSG21 & $\mathrm{A} 3$ & $\mathrm{~A} 3$ & $\mathbf{A 3}$ \\
\hline $\mathrm{A} 2$ & $\mathrm{~A} 3$ & $\mathbf{A 3}$ & FSG31 & $\mathrm{A} 2$ & $\mathrm{~A} 3$ & $\mathbf{A 3}$ \\
\hline $\mathrm{A} 2$ & $\mathrm{~A} 3$ & A3 & SSC32 & $\mathrm{A} 3$ & $\mathrm{~A} 3$ & $\mathbf{A 3}$ \\
\hline $\mathrm{A} 2$ & $\mathrm{~A} 3$ & $\mathbf{A 3}$ & FST21 & $\mathrm{N}$ & $\mathrm{A} 3$ & $\mathbf{N}$ \\
\hline $\mathrm{A} 2$ & $\mathrm{~A} 3$ & A3 & FST22 & $\mathrm{N}$ & $\mathrm{A} 3$ & $\mathbf{N}$ \\
\hline $\mathrm{N}$ & $\mathrm{A} 3$ & $\mathbf{N}$ & FUL62 & $\mathrm{N}$ & $\mathrm{A} 3$ & $\mathbf{N}$ \\
\hline $\mathrm{A} 2$ & $\mathrm{~A} 3$ & $\mathbf{A 3}$ & SSC33 & $\mathrm{A} 3$ & $\mathrm{~A} 3$ & $\mathbf{A 3}$ \\
\hline $\mathrm{A} 2$ & A3 & A3 & FSB23 & $\mathrm{A} 2$ & $\mathrm{~A} 3$ & $\mathbf{A 3}$ \\
\hline $\mathrm{A} 1$ & $\mathrm{~A} 3$ & A3 & FSG23 & $\mathrm{A} 3$ & $\mathrm{~A} 3$ & $\mathbf{A 3}$ \\
\hline $\mathrm{A} 2$ & $\mathrm{~A} 3$ & A3 & FST23 & $\mathrm{N}$ & $\mathrm{A} 3$ & $\mathbf{N}$ \\
\hline $\mathrm{N}$ & $\mathrm{A} 3$ & $\mathbf{N}$ & FUL63 & $\mathrm{N}$ & $\mathrm{A} 3$ & $\mathbf{N}$ \\
\hline $\mathrm{A} 3$ & $\mathrm{~A} 3$ & $\mathbf{A 3}$ & FSC54 & $\mathrm{A} 3$ & $\mathrm{~A} 3$ & $\mathbf{A 3}$ \\
\hline $\mathrm{A} 1$ & $\mathrm{~A} 3$ & $\mathbf{A 3}$ & FSG24 & $\mathrm{A} 3$ & $\mathrm{~A} 3$ & $\mathbf{A 3}$ \\
\hline $\mathrm{A} 2$ & $\mathrm{~A} 3$ & A3 & FSG34 & $\mathrm{A} 2$ & $\mathrm{~A} 3$ & $\mathbf{A 3}$ \\
\hline $\mathrm{A} 2$ & A3 & A3 & FSS14 & $\mathrm{A} 2$ & A3 & $\mathbf{A 3}$ \\
\hline $\mathrm{A} 2$ & A3 & A3 & FST24 & $\mathrm{N}$ & A3 & $\mathbf{N}$ \\
\hline $\mathrm{N}$ & A3 & $\mathbf{N}$ & FUL64 & $\mathrm{N}$ & A3 & $\mathbf{N}$ \\
\hline $\mathrm{A} 1$ & A3 & A3 & FSG25 & $\mathrm{A} 3$ & A3 & $\mathbf{A 3}$ \\
\hline $\mathrm{A} 2$ & A3 & $\mathbf{A 3}$ & FST25 & $\mathrm{N}$ & A3 & $\mathbf{N}$ \\
\hline $\mathrm{A} 2$ & A3 & A3 & FSG35 & $\mathrm{A} 2$ & A3 & $\mathbf{A 3}$ \\
\hline $\mathrm{A} 2$ & $\mathrm{~A} 3$ & $\mathbf{A 3}$ & FSS15 & $\mathrm{A} 2$ & $\mathrm{~A} 3$ & $\mathbf{A 3}$ \\
\hline $\mathrm{A} 1$ & $\mathrm{~A} 3$ & A3 & FSG26 & $\mathrm{A} 3$ & $\mathrm{~A} 3$ & $\mathbf{A 3}$ \\
\hline $\mathrm{A} 2$ & $\mathrm{~A} 3$ & $\mathbf{A 3}$ & FSG36 & $\mathrm{A} 2$ & $\mathrm{~A} 3$ & $\mathbf{A 3}$ \\
\hline $\mathrm{A} 2$ & $\mathrm{~A} 3$ & A3 & FST26 & $\mathrm{N}$ & $\mathrm{A} 3$ & $\mathbf{N}$ \\
\hline $\mathrm{A} 2$ & $\mathrm{~A} 3$ & $\mathbf{A 3}$ & SSC37 & $\mathrm{A} 3$ & $\mathrm{~A} 3$ & $\mathbf{A 3}$ \\
\hline $\mathrm{A} 2$ & $\mathrm{~A} 3$ & A3 & FST27 & $\mathrm{N}$ & $\mathrm{A} 3$ & $\mathbf{N}$ \\
\hline $\mathrm{N}$ & $\mathrm{A} 3$ & $\mathbf{N}$ & FUL67 & $\mathrm{N}$ & $\mathrm{A} 3$ & $\mathbf{N}$ \\
\hline $\mathrm{A} 2$ & A3 & A3 & SSC38 & $\mathrm{A} 3$ & $\mathrm{~A} 3$ & $\mathbf{A 3}$ \\
\hline $\mathrm{A} 2$ & $\mathrm{~A} 3$ & A3 & FST28 & $\mathrm{N}$ & $\mathrm{A} 3$ & $\mathbf{N}$ \\
\hline $\mathrm{N}$ & $\mathrm{A} 3$ & $\mathbf{N}$ & FUL68 & $\mathrm{N}$ & $\mathrm{A} 3$ & $\mathbf{N}$ \\
\hline A3 & $\mathrm{A} 3$ & A3 & FSC59 & $\mathrm{A} 3$ & $\mathrm{~A} 3$ & $\mathbf{A 3}$ \\
\hline $\mathrm{A} 2$ & A3 & A3 & FSS19 & $\mathrm{A} 2$ & A3 & $\mathbf{A 3}$ \\
\hline $\mathrm{A} 2$ & A3 & A3 & FSG39 & $\mathrm{A} 2$ & A3 & $\mathbf{A 3}$ \\
\hline $\mathrm{A} 2$ & A3 & A3 & FST29 & $\mathrm{N}$ & A3 & $\mathbf{N}$ \\
\hline $\mathrm{N}$ & A3 & $\mathbf{N}$ & FUL69 & $\mathrm{N}$ & A3 & $\mathbf{N}$ \\
\hline $\mathrm{A} 2$ & A3 & $\mathbf{A 3}$ & SSC310 & A3 & A3 & $\mathbf{A 3}$ \\
\hline $\mathrm{A} 1$ & A3 & $\mathbf{A 3}$ & FSG210 & A3 & A3 & $\mathbf{A 3}$ \\
\hline $\mathrm{A} 2$ & A3 & A3 & FSG310 & $\mathrm{A} 2$ & A3 & $\mathbf{A 3}$ \\
\hline $\mathrm{A} 2$ & $\mathrm{~A} 3$ & $\mathbf{A 3}$ & FST210 & $\mathrm{N}$ & $\mathrm{A} 3$ & $\mathbf{N}$ \\
\hline $\mathrm{N}$ & $\mathrm{A} 3$ & $\mathbf{N}$ & FUL610 & $\mathrm{N}$ & $\mathrm{A} 3$ & $\mathbf{N}$ \\
\hline $\mathrm{A} 2$ & $\mathrm{~A} 3$ & A3 & SSC311 & $\mathrm{A} 3$ & $\mathrm{~A} 3$ & $\mathbf{A 3}$ \\
\hline $\mathrm{A} 1$ & $\mathrm{~A} 3$ & A3 & FSG211 & $\mathrm{A} 3$ & $\mathrm{~A} 3$ & $\mathbf{A 3}$ \\
\hline $\mathrm{A} 2$ & $\mathrm{~A} 3$ & A3 & FST211 & $\mathrm{N}$ & $\mathrm{A} 3$ & $\mathbf{N}$ \\
\hline $\mathrm{A} 2$ & A3 & A3 & FSG311 & $\mathrm{A} 2$ & A3 & $\mathbf{A 3}$ \\
\hline $\mathrm{A} 2$ & A3 & A3 & SSC312 & $\mathrm{A} 3$ & $\mathrm{~A} 3$ & $\mathbf{A 3}$ \\
\hline $\mathrm{A} 2$ & A3 & A3 & FST212 & $\mathrm{N}$ & A3 & $\mathbf{N}$ \\
\hline $\mathrm{N}$ & $\mathrm{A} 3$ & $\mathbf{N}$ & FUL612 & $\mathrm{N}$ & A3 & $\mathbf{N}$ \\
\hline $\mathrm{A} 2$ & A3 & A3 & SSC313 & A3 & A3 & $\mathbf{A 3}$ \\
\hline $\mathrm{A} 2$ & A3 & $\mathbf{A 3}$ & FSB213 & $\mathrm{A} 2$ & A3 & $\mathbf{A 3}$ \\
\hline A3 & A3 & A3 & FSC513 & A3 & A3 & $\mathbf{A 3}$ \\
\hline A1 & A3 & $\mathbf{A 3}$ & FSG213 & $\mathrm{A} 3$ & A3 & $\mathbf{A 3}$ \\
\hline $\mathrm{A} 2$ & A3 & $\mathbf{A 3}$ & FST213 & $\mathrm{N}$ & A3 & $\mathbf{N}$ \\
\hline $\mathrm{A} 2$ & A3 & $\mathbf{A 3}$ & FSG313 & $\mathrm{A} 2$ & A3 & $\mathbf{A 3}$ \\
\hline $\mathrm{A} 2$ & $\mathrm{~A} 3$ & $\mathbf{A 3}$ & FSS113 & $\mathrm{A} 2$ & $\mathrm{~A} 3$ & $\mathbf{A 3}$ \\
\hline $\mathrm{N}$ & A3 & $\mathbf{N}$ & FUL613 & $\mathrm{N}$ & A3 & $\mathbf{N}$ \\
\hline
\end{tabular}




\section{Conclusiones}

- La región estudiada presenta una mayor aptitud para el establecimiento de la especie laurel de cera que para la uva camarona, destacándose la presencia de una superficie importante, aproximadamente 6.000 hectáreas, con un alto grado de aptitud (A1), localizada principalmente en los municipios de Suesca, Chocontá, Sesquilé y Villapinzón.

- Existe una superficie importante con aptitud moderada (A2) para la implementación de laurel de cera $(56 \%)$ y de uva camarona (35\%), lo que muestra una significativa perspectiva regional en la posibilidad de llevar a cabo programas agrarios sostenibles que puedan reorientar el inadecuado uso actual de la tierra, complementados con la opción de adelantar mejoramientos sobre algunas de las zonas con problemas ambientales y socioeconómicos actuales.

- Particularmente en el municipio de Villapinzón, el $94 \%$ de su superficie presenta aptitud entre alta (A1) a moderada (A2) para la implementación de laurel de cera, principalmente en las veredas Chasquez y Quincha y el $25 \%$ de su superficie con aptitud moderada (A2) para uva camarona, para lo cual la vereda Guanguita representa la mejor opción.
- En relación con las variables socioeconómicas consideradas, los problemas asociados con el acceso al crédito, el bajo nivel de escolaridad y en algunos casos el uso de la tierra, constituyen las limitantes más importantes que afectan el grado de aptitud integral del municipio.

- Aunque las decisiones sobre la planificación del uso de la tierra se apoyan en gran medida en los aspectos biofísicos y económicos, es el comportamiento de los humanos el que presenta mayor influencia, razón por la cual en las evaluaciones sobre el mejor uso de la tierra se debe contemplar el componente social de una manera más definitiva y concreta, lo que en este momento plantea el mayor desafío metodológico que debe ser superado con el levantamiento de una información más confiable y sistemática al respecto.

- Con el propósito de incorporar un valor agregado a los productos obtenidos mediante los tipos de utilización aquí planteados, se debe contemplar el desarrollo de procesos agroindustriales que permitan a los pobladores obtener mejores ingresos, conducentes a alcanzar un mejor nivel de vida y por ende, un mejor desarrollo de la región. 


\section{Bibliografía}

ABUBAKR, Mohamed; SHARIFI, Mohamed and KEULEN, Herman. An integrated agro-economic and agro-ecological methodology for land use planning and policy analysis. En: International journal of aerospace survey and earth sciences: Revista del International Institute for Aerospace Survey and Earth Sciences. I.T.C. Issue 2, Enschede. 2000. pp. 87-94.

ACERO, Luis Enrique. Guía para el cultivo, aprovechamiento y conservación de la Uva camarona (Macleania rupestris). Bogotá: Convenio Andrés Bello. 2001. 28 p.

ANDRADE, Angela. Marco teórico y metodológico para la planificación del uso de las tierras con énfasis en aspectos forestales. En: Revista informativa del proyecto SIG-PAFC. Año $1, \mathrm{~N}^{\circ} 2$ (mayo 1994). pp. 22-26.

ANDRADE, Angela y GONZÁLEZ, Armando. Aspectos conceptuales y metodológicos para el diseño de las bases de datos utilizados en el análisis de los sistemas de producción. En: Revista informativa del proyecto SIG-PAFC. Año 3, $\mathrm{N}^{\circ} 10-11$ (sep. 1996). pp. 88-166.

BABTISPE, Luis et al. Bases para un plan de desarrollo regional de las provincias de Norte y Gutiérrez (Boyacá). En: Ambiente y Desarrollo. Revista del Instituto de Estudios Ambientales para el Desarrollo de la Universidad Javeriana. Año 1, $\mathrm{N}^{\circ} 1$ (sep. 1993); pp. 91-120.

BOTERO, Pedro. Aproximación conceptual a la Zonificación Ambiental, caso Apaporis-Tabatinga. En : Propuesta metodológica para zonificación ecológica-económica para la Amazonía. Memorias del Seminario Taller Santafé de Bogotá. Tratado de Cooperación Amazónica. Lima, Perú. (dic. 1996); pp. 170-194.

CASTRO, Juan Carlos y FORERO, Camilo. Propagación vegetativa y por semilla de uva camarona (Macleania rupestris). Bogotá, 1994, 98 p. Trabajo de grado (Ingeniero Forestal). Universidad Distrital Francisco José de Caldas. Facultad del Medio Ambiente y Recursos Naturales. Proyecto Curricular de Ingeniería Forestal.

Centro Interamericano de Fotointerpretación. Metodología para delimitación de áreas homogéneas. Bogotá. 1981. 261 p.

Colombia. Corporación de la defensa de la meseta de Bucaramanga. Guía de reforestación. Bogotá: La Corporación, 1989. 213 p.

Colombia. Gobernación de Cundinamarca. Anuario Estadístico. Bogotá: 1999.

54 Fernando Rodríguez Mojica 
Colombia. Ministerio del Medio Ambiente - Instituto Geográfico Agustín Codazzi. Zonificación Ecológica de la Región Pacífica Colombiana. Bogotá: 2000. 365 p.

Convenio Andrés Bello. Especies Vegetales Promisorias de los países del Convenio. CAF. Tomo XII. Bogotá. 1998.

DE LAS SALAS, Gonzalo. Suelos y Ecosistemas Forestales con énfasis en América Tropical. Costa Rica: IICA, 1987. 445 p.

Esquema de Ordenamiento Territorial del municipio de Villapinzón: Componente rural. Villapinzón. 2001. 76 p.

ETTER, Andrés. Introducción a la Ecología del Paisaje: Un marco de integración para los levantamientos rurales. Instituto Geográfico Agustín Codazzi. Subdirección de docencia e investigación. Unidad de levantamientos rurales. Bogotá. 1991. 88 p.

Instituto Geográfico Agustín Codazzi. Subdirección de Geografía. Guía Metodológica para la formulación del Plan de Ordenamiento Territorial Municipal. Bogotá, 1997. 185 p.

Subdirección de Geografía. Zonificación Socioeconómica de la Cuenca hidrográfica del río Sinú. Bogotá, 1998. 140 p. Bogotá: 2001.

Subdirección de Catastro. Estadísticas Catastrales Rurales para el municipio de Villapinzón.

LEON PEREZ, Jonás. Metodología para la zonificación forestal. En: Aproximación a la definición de criterios para la zonificación y ordenamiento forestal en Colombia. Bogotá: Inderena, 1994; pp. 83-107.

MACHADO, Absalón. La cuestión agraria en Colombia a fines del milenio. 1 ed. Bogotá: El Ancora Editores, 1998. 222 p.

MALAGON, Ester y USSA, Jaime. Implementación y determinación de la producción inicial de un módulo agropecuario para el piso alto Andino. Municipio de Cogua. Bogotá, 2000, 130 p. Trabajo de grado (Ingeniero Forestal). Universidad Distrital Francisco José de Caldas. Facultad del Medio Ambiente y Recursos Naturales. Proyecto Curricular de Ingeniería Forestal.

MARTINEZ, Sandra; PATIÑO, Zaida y SANCHEZ, Olga. Valoración del aspecto social en la evaluación del uso de la tierra: Propuesta Metodológica. En : Revista Perspectiva Geográfica: Revista del Programa de Estudios de Posgrado en Geografía de la Universidad Pedagógica y Tecnológica de Colombia. $\mathrm{N}^{\circ}$ 6. (2001); pp.177-205.

MENDEZ, Elías. Planificación Ambiental y Desarrollo Sostenible. Centro Interamericano de Desarrollo e Investigación Ambiental y Territorial. Mérida, Venezuela. 1996. 144 p.

MONTAÑEZ, Gustavo. ¿Hacia dónde va la Sabana de Bogotá? Bogotá: Editorial Sena. CES, Universidad Nacional. 1992. 373 p.

MORA, Luis y STURM, Helmut. Estudios ecológicos del páramo y del bosque altoandino en la cordillera oriental de Colombia. Bogotá: Academia Colombiana de Ciencias Exactas, Físicas y Naturales. Tomo II. 1994. 361 p. 
MUÑOZ, Jairo y LUNA, Cristina. Guía para el cultivo, aprovechamiento y conservación del Laurel de cera (Myrica pubescens). Bogotá: Convenio Andrés Bello. 1999. 36 p.

Determinación de algunas características de la semilla del Laurel de cera, obtenida en el municipio de San José de Albán. Departamento de Nariño. En: Revista de Ciencias Agrícolas. Universidad de Nariño. (2000). pp. 34-48.

Organización de las Naciones Unidas para la Agricultura y la Alimentación. FAO. Esquema para la evaluación de tierras. Roma. 1976. 66 p.

Organización de las Naciones Unidas para la Agricultura y la Alimentación. FAO. Evaluación de tierras con fines forestales. Roma.1984. 105 p.

Organización de las Naciones para la Agricultura y la Alimentación. FAO. Directivas : Evaluación de Tierras para Agricultura de Secano. Roma. 1985. 228 p.

OSSIO, Hernán. Propuesta metodológica de zonificación ecológica-económica de Bolivia. En: Propuesta metodológica para zonificación ecológica-económica para la Amazonía. Memorias del Seminario Taller Santafé de Bogotá. Tratado de Cooperación Amazónica. Lima, Perú. (Dic. 1996); pp. 213-233.

RICHTERS, Eric. Manejo del uso de la tierra en América Central: Hacia el aprovechamiento sostenible del recurso tierra. Costa Rica; IICA, 1995. 439 p.

Sociedad Geográfica de Colombia. Cuenca alta del río Bogotá: Región Programa. Plan de Ordenamiento Territorial. Bogotá. 2000. 144 p.

STURM, H y Rangel, O. Ecología de los páramos Andinos. Una visión preliminar integrada. $\mathrm{N}^{\circ}$ 9: Instituto de Ciencias Naturales. Universidad Nacional. Bogotá. 1985. 292 p.

UTRIA, Rubén. Ordenamiento Territorial: Hacia un enfoque conceptual. En: memorias Taller sobre Ordenamiento Territorial, conceptualizaciones y orientaciones. Instituto Geográfico Agustín Codazzi. Bogotá; pp. 41-52.

La Metropolización de Bogotá y la Sabana. Cuadernos de la Sociedad Colombiana de Planificación. $\mathrm{N}^{\circ}$ 1. (2000). 105 p.

VILLOTA, Hugo. Una nueva aproximación a la clasificación fisiográfica del terreno. En: Revista CIAF. Volumen 15, N 1. Bogotá. 1997. pp. 83-99.

56 Fernando Rodríguez Mojica 